\title{
Proactividad y resiliencia en estudiantes emprendedores de Lima
}

\section{Proactivity and Resilience in Entrepreneurial Students of Lima}

Jhon Alexander Holguin Alvarez*(D)

Universidad César Vallejo, Lima, Perú

ORCID: https://orcid.org/0000-0001-5786-0763

Marcos Fabris Rodríguez Castillo

Institución Educativa Privada San Nicolás, Lima, Perú

ORCID: https://orcid.org/0000-0002-9125-4457

Recibido 07-09-18 Revisado 21-11-18 Aprobado 07-05-19 En línea 30-07-19

*Correspondencia

Email: jhajazzy@gmail.com
Citar como:

Holguin Alvarez, J., \& Rodríguez Castillo, M. (2020). Proactividad y resiliencia en estudiantes emprendedores de Lima. Propósitos y Representaciones, 8(2), e367. doi: http://dx.doi.org/10.20511/pyr2020.v8n2.367 


\section{Resumen}

Los avances en el estudio sobre resiliencia han reportado hasta el momento, indicios de características emergentes de proactividad en estudiantes que se profesionalizan, y a su vez, se embarcan en alguna actividad laboral como visionarios de su superación personal y profesional, esto desencadenó su estudio, con el propósito de determinar la relación entre resiliencia y proactividad de 285 estudiantes de Instituciones de Educación Superior de seis distritos de Lima $(\chi=32.7$ años de edad), estos desarrollaban alguna actividad laboral de modo formal o informal, como instrumentos de medición se utilizaron la Escala de Comportamiento proactivo en las organizaciones y la Escala de Resiliencia; se concluyó en la existencia de relación intensa y positiva entre la resiliencia y proactividad de los estudiantes evaluados, fue mayor la relación en aquellos que recibieron apoyo económico familiar durante su profesionalización, a su vez, los emprendedores más proactivos son aquellos que desarrollan actividades laborales informales, los emprendedores tanto formales como informales presentan niveles de resiliencia similares; finalmente, la contribución de esta investigación permitió verificar que el pensamiento visionario estimula los objetivos del ser emprendedor como los del ser profesional como competencias humanísticas formativas sociales.

Palabras clave: Formación del carácter; Formación profesional superior; Proactividad; Resiliencia; Trabajador independiente.

\section{Summary}

The advances in the study on resilience have reported so far, indications of emerging characteristics of proactivity in students who are professionalized, and in turn, embark on some work activity as visionaries of their personal and professional improvement, this triggered their study, with The purpose of determining the relationship between resilience and proactivity of 285 students of Higher Education Institutions of six districts of Lima $(\chi=32.7$ years of age), these developed some formal or informal work, as measurement instruments were used Scale of proactive behavior in organizations and the Resilience Scale; it was concluded in the existence of an intense and positive relationship between the resilience and proactivity of the students evaluated, the relationship was greater in those who received family economic support during their professionalization, in turn, the most proactive entrepreneurs are those who develop informal work activities, both formal and informal entrepreneurs present similar levels of resilience; Finally, the contribution of this research allowed us to verify that visionary thinking stimulates the objectives of the entrepreneurial being as those of the professional being as humanistic social formative competences.

Keywords: Formation of the Character; Higher Professional Formation; Proactivity; Resilience; Independent Worker.

\section{Introducción}

Los emprendedores que tienen como meta llegar a ser microempresarios formales, crecen con mucho esfuerzo, su capacidad se fortalece acorde a las necesidades y exigencias del entorno social. En el caso de Lima, estos afrontan una lucha diaria generada por un condicionante: la competencia económica provocada por los consumidores, que, aunque en algunos casos es combatida por la sinergia del compañerismo en cualquier empresa, dicha competencia suele ser intensa, sobre todo en emprendedores que conforman microempresas vírgenes ${ }^{l}$, como también en aquellas que llevan, por lo menos, más de cinco años en cualquier tipo de comercio, estos suelen superar obstáculos constantes, indudablemente por un tema que les preocupa: la inversión. En 
otro lugar, no menos importante, se encuentran la familia, cuyo sostén espiritual alimenta la superación en dichos empresarios. Finalmente, todo es mucho más difícil cuando estos empresarios deciden tomar el camino de la profesionalización en alguna institución de educación superior peruana privada o pública.

En Lima, muchos estudiantes de instituciones de educación superior afrontan necesidades económicas, buscan soluciones, pero en ese proceso suelen aparecer eventos vitales: enamoramiento, decepción, frustración académica, pero, si en esos aparece la perdida familiar, entonces la resiliencia llegaría al clímax si el sujeto logra superar dicha pena para continuar con el triunfo comercial añorado, en el caso de alumnos becarios la exigencia académica recae en aquellos del tercio superior, y entre los cuales las oportunidades de trabajo son más exigentes (Faúndez et al., 2017; Price \& Sedlak, 2018), y este discurso suele aparecer en estudiantes resilientes que enfrentan diferencias sociales pero tratan de dar más de sus competencias profesionales (Adejei, Dontoh \& Baafi-Frimpong, 2017; Bradshaw et al., 2018; Cabrera, 2015; Chafloque et al., 2018; Pereira, 2016; Simón, 2017; Stanley et al., 2015), otros enfrentan las dificultades netamente cognitivas (Quijada, 2018; Velásquez-Acosta, 2017) y otros por pertenecer a contextos vulnerables (Silva \& Jiménez, 2015; Simón, 2017), como también por necesidades económicas en el contexto latinoamericano (Atoche, 2017; Quijada, 2018, Velásquez-Acosta, 2017); y algunos estudiantes se sienten satisfechos con la vida pero otro grupo no lo es por motivos académicos (Adjei, Dontoh, \& Baafi-Frimpong, 2017; Guevara, 2017; Pereira, 2016; Velásquez-Acosta, 2017) y otros, hasta enfrentan distintos tipos de exclusión laboral determinadas por indicios de racismo (Bello, 2013).

Sin embargo, uno los eventos con mayor potencia devastadora para los emprendedores es la muerte en sus distintas expresiones, solo para tratar una de ellas, el índice de homicidios en el Perú reportado por el Instituto Nacional de Estadística e Informática - INEI (2017), se incrementó a más de un punto porcentual desde el año $2011(5,4)$ hasta el año $2016(7,2)$ en Lima; y entre los distritos que sobresalen Los Olivos (12,4), Ventanilla (8,3), San Martín de Porres (8,1), Comas (7,5), San Juan de Lurigancho (7,1), Puente Piedra (6,1). Entre estas cifras, muchos de los estudiantes universitarios que se dedican al comercio formal o informal en distintos rubros (alimenticios y de ocio), como aquellos trabajadores de empresas privadas de transporte, suelen perderlo todo cuando este tipo de eventos se suscita en sus vidas. En cuanto al emprendedor empresarial, este siente el fracaso en dicho momento, o pueden suscitarse otros riesgos como el robo a mano armada, raptos o cobros por cupos de mafias locales; y como resultado pierden grandes fracciones de inversión monetaria. Esto sugiere desarrollar una competencia humanística: la resiliencia, lo que los ayudará a superar ante cualquier evento como estos, uno de ellos originaria un cambio cognitivo y actitudinal en sus vidas de negocio, de otro modo, es necesario que aflore el comportamiento proactivo como medio de superación para mantener indemnes los objetivos de su autorrealización como microempresario.

Esta capacidad de superación resiliente ya es una base humana de gran competencia para las empresas en la actualidad, ya que permite la toma de decisiones y la formulación estrategia de una inversión efectiva auto generadora de recursos (De Souza \& Machado, 2013), por lo que para un empresario iniciado, también es un factor del desarrollo de su proactividad si es que desea generar una línea de desarrollo desde su capacidad emprendedora, en otro caso, al recurrir a la informalidad, el inversionista puede invertir con mayor predisposición como una imitación de los procesos del sector formalizado (Zarate-Hoyos \& Larios-Meoño, 2015), no obstante, si la remuneración incrementa la proactividad y su perdurabilidad (Caraballo, 2015; Ortiz, 2016), e inclusive la toma de decisiones que favorece dicha proactividad genera estabilidad y sostenibilidad del trabajo continuo (Polanco, Ramírez \& Orozco, 2016) o la sobrevivencia para la superación con mejores competencias emocionales en el emprendedor (Garrosa-Hernández, et al., 2013; Moreno-Jiménez \& Gálvez, 2013; Peraza, Gómez \& Aleixandre, 2016; Torrente, Salanova \& Llorens, 2013). En cuanto a sus habilidades efectivas, la proactividad permite que los sujetos resilientes desarrollen características importantes para dicha perdurabilidad como empresarios o trabajadores dependientes, entre los cuales son funcionales (Ávalo, Yagüe \& 
Cangahuala, 2016; Olea-Miranda, Contreras \& Barcelo- Valenzuela, 2016; Redchenko, 2016): la interacción comunicativa exitosa, motivación hacia la satisfacción de necesidades intrínsecas, la planificación adaptativa, estructuración de propósitos funcionales, y transferencia de conocimientos.

\section{Las competencias humanísticas: proactividad y resiliencia.}

La proactividad es una capacidad formativa del carácter (Covey, 1996; Covey, 2007), fortalecida como actitud mediante la autoevaluación, toma de decisiones y la formación del lenguaje proactivo para el alcance de metas de forma progresiva (Dehnad, 2017; Xifra, 2009). Sin embargo, su fortalecimiento es permanente en cuanto el ser humano alcance determinado grado de éxito, ya sea profesional o personal. Alguna parte de su evidencia formativa alude a la proactividad como una capacidad de autodominio (Bradshaw et al., 2018; Geertshuis, Jung \& Cooper-Thomas, 2014; Keskinkilic-Kara \& Zafer-Gunes, 2017), y se desarrolla por aspectos disciplinares del comportamiento cultural, y el crecimiento de actitudes puestas en práctica para la adaptabilidad y el compromiso laboral (Bradshaw et al., 2018; Larson et al., 2018; Pennaforte, 2016; Salessi \& Omar, 2017).

Holguin (2017) reportó que las estrategias en formación proactiva eran más efectivas que la formación prosocial en estudiantes con cierta reactancia escolar, ya que ciertas características implicaban incidentes críticos y posibilidades de generar estresores sociales. Esto se extrapola a sujetos con capacidades para superar el estrés en sus labores de forma organizativa, por ende, mediante situaciones proactivas que no necesariamente implican una perdida, sino más bien, alta exigencia para su superación y ser más competitivos (Fernandez-Ronquillo, Llinas-Audet \& Ferran-Sabate, 2018; Lámbarry, Trujillo \& Cumbres, 2016; Salessi \& Omar, 2017).

La resiliencia se considera como un constructo social, adoptado en situaciones adversas y otros sujetos potencialmente resilientes adquieren por un andamiaje cognitivo y de adopción consciente o inconsciente (Cyrulnik, 2016; Ungar, 2008; 2012; West, 2005), aunque el constructo también se ha establecido como la característica psicológica apremiante en momentos de desesperación y frustración (Cyrulnik, 2016; Fletcher \& Sarkar, 2013). No obstante, hallazgos más comunes sugieren que la interrelación social como las formas de afrontamiento y su desarrollo proactivo se impliquen en el estudio de su desarrollo o logro humano (Fletcher \& Sarkar, 2013; Gaxiola et al. 2013), es decir, cómo los sujetos resuelven la presencia de un obstáculo más que aceptar y superarlo, y en qué factores se valen para realizar dichos procesos (Didkowsky, Fletcher \& Sarkar, 2013; Ungar \& Liebenberg, 2010; Ungar, 2013; 2014), sin embargo, es importante para la sociedad de hoy conservar niveles ideológicos de desarrollo: a) ontogenético (individual), b) micro sistémico (familias-instituciones), c) meso sistémico (gruposorganizaciones), d) exo sistémico (estructural sociales), e) macro sistémico (ideológico), f) crono sistémico (eventos paradigmáticos) (West, 2005, p. 47).

Las evidencias científicas del estudio de resiliencia (Chen, 2016), describen que estudiantes chinos con determinada personalidad resiliente afrontan exigencias académicas y personales para ser exitosos, lo cual sucede en otras poblaciones académicas (Brouskeli, Kaltsi y Loumakou, 2018). El estudio de Dutra-Thomé, Siqueira y Koller (2016), demostró que los estudiantes que aspiran el desarrollo laboral necesitan mayor preparación psicológica para abordar exigencias académicas durante el proceso de desarrollo; esto obedece a la teoría de liderazgo para el éxito resiliente (Ozmusul, 2017), la cual exigen que se desarrollen la mentalidad resiliente, emocionalidad, sociabilidad, ética y aptitudes físicas resilientes. Sin embargo, en la universidad son esenciales el desarrollo de los valores que acompañen la culturización y maduración de la resiliencia (Stanley et al., 2015). Algo muy importante cabe señalar, desde el estudio de Galiana (2015), se puede considerar que el optimismo, felicidad y la actitud hacia los desafíos se han convertido en algunos de los elementos que fortalecen la resiliencia ya sea para un desempeño en el estudio o para la proactividad laboral. 
Parte de estos hallazgos se relacionan a algunos problemas a nivel nacional en estudiantes universitarios: de tipo psicológico (estrés, desesperación y nublación), o a los de tipo personal académico (ausentismo, deserción, procrastinación) (Cabrera, 2015; Chafloque, 2018). Estos suelen aparecer entre los estudiantes universitarios que inician su carrera universitaria o en aquellos que están a punto de egresar. Ahora bien, en cuanto a los microempresarios que se inician, surgen problemas como que no cumplan con reglas o requisitos para formalizarse en las Municipalidades respectivas, de los cuales, existen dos casos marcados: aquellos que trabajan de forma independiente con inversión propia de la empresa (menor a 10 trabajadores a cargo) (Superintendencia Nacional de Aduanas y de Administración Tributaria-SUNAT, 2018) y los dependientes, que trabajan para empresas conformadas por otros sujetos de inversión. En el caso de esta investigación, el estudio fijó su atención en el caso de los microbuseros y cobradores informales como primer caso, y otro, desde el estudio son los emolienteros, vendedores de alimentos, implementos de limpieza y recursos para el ocio.

Este último grupo, si bien si cumple con ciertos requisitos para ventas de productos, también sus integrantes se profesionalizan al igual que los vendedores de artículos de limpieza y de recreación (venta de celulares, videos, entre otros). Todo lo vertido hasta aquí permitió abordar e intentar dar respuesta a los siguientes cuestionamientos: a) ¿Un análisis relacional entre proactividad y resiliencia será significativo en estudiantes de educación superior de acuerdo a su condición de emprendedores: con apoyo familiar (sin perdida familiar) y sin apoyo familiar (con perdida familiar)?, b) ¿esta relación será funcional por cada distrito abordado?; y c) ¿es mayor la proactividad o la resiliencia en los emprendedores formales e informales?

El estudio tiene por propósito: analizar la relación entre proactividad en estudiantes universitarios microempresarios con y sin requisitos para comercializar, los cuales viven y laboran en seis distritos de la ciudad de Lima: San Juan de Lurigancho, Comas, Puente Piedra, San Martín de Porres, Los Olivos y Ventanilla.

\section{Método}

La investigación es de enfoque cuantitativo transeccional (Hernández, Fernández \& Baptista, 2014), y se realizó mediante un estudio correlacional y otro comparativo (Hernández et al., 2014; Sabino, 2014); esto en razón al propósito de analizar la relación entre la proactividad y resiliencia, como también compararlas por cada grupo de estudiantes de educación superior que emprendieron algún ejercicio laboral formal o informal durante los últimos tres a cinco años próximos al estudio.

\section{Participantes}

El estudio contó con una muestra de 285 estudiantes de Instituciones de Educación Superior privada y pública, Centros de Educación Técnica Productiva; como también de Universidades de gestión pública y privada de los distritos San Juan de Lurigancho, Comas, Puente Piedra, San Martín de Porres, Los Olivos y Ventanilla, en la ciudad de Lima (65\% de género masculino; 35 $\%$ del género femenino; $\chi=32.7$ años). El muestreo fue no probabilístico de tipo conveniencia, esto debido a la accesibilidad a los estudiantes por cada universidad, y que fuesen diferenciados por las características deseables para el estudio y su especificidad (tabla 1), fue un limitante aunado al factor económico y tiempo para la realización del estudio, y se tuvo acceso a estudiantes de cuatro tipos de institución educativa superior correspondientes a seis distritos de Lima (tabla 2). En cuanto a los criterios de selección, todos los participantes realizaban una actividad laboral: a) algunos como trabajadores dependientes de alguna empresa, o b) eran trabajadores independientes, los cuales se habían forjado durante tres años antes al estudio como emprendedores iniciados. El criterio de selección para inclusión en el estudio se diferenció por informalidad versus formalidad (tabla 1). 
Tabla 1.

Características y criterios de selección de participantes en el estudio de acuerdo a informalidad y formalidad

\begin{tabular}{|c|c|c|c|}
\hline \multicolumn{2}{|c|}{ Emprendedor dependiente } & \multicolumn{2}{|c|}{ Emprendedor independiente } \\
\hline Formal $(*)$ & Informal $(* *)$ & Formal (\#) & Informal (\#\#) \\
\hline $\begin{array}{l}\text { Mozo de Restaurant } \\
\text { con Registro Público }\end{array}$ & Vendedor de comida & $\begin{array}{l}\text { Conductor en } \\
\text { transporte } \\
\text { privado (microbús) }\end{array}$ & $\begin{array}{l}\text { Conductor de } \\
\text { transporte } \\
\text { privado (microbús) } \\
\text { Conductor de }\end{array}$ \\
\hline Chef & $\begin{array}{l}\text { Mozo de venta de } \\
\text { comida rápida }\end{array}$ & Emolientero & $\begin{array}{l}\text { transporte } \\
\text { privado (taxi) }\end{array}$ \\
\hline $\begin{array}{l}\text { Conductor en } \\
\text { transporte } \\
\text { público (microbús) }\end{array}$ & Ayudante de cocina & $\begin{array}{l}\text { Vendedor asociado de } \\
\text { comida }\end{array}$ & Cobrador de microbús \\
\hline $\begin{array}{l}\text { Jefe o coordinador de } \\
\text { tienda }\end{array}$ & $\begin{array}{l}\text { Vendedor de artículos } \\
\text { de limpieza }\end{array}$ & $\begin{array}{l}\text { Vendedor asociado de } \\
\text { celulares }\end{array}$ & Emolientero \\
\hline Reponedor & $\begin{array}{l}\text { Vendedor de video } \\
\text { DVD/Cd }\end{array}$ & $\begin{array}{l}\text { Vendedor de periódico } \\
\text { en módulo }\end{array}$ & $\begin{array}{l}\text { Vendedor de periódico } \\
\text { sin módulo }\end{array}$ \\
\hline Cajero & $\begin{array}{l}\text { Vendedor de } \\
\text { alimentos }\end{array}$ & $\begin{array}{l}\text { Vendedor de } \\
\text { alimentos } \\
\text { en mercado }\end{array}$ & $\begin{array}{l}\text { Vendedor de video } \\
\text { DVD/Cd }\end{array}$ \\
\hline Otros & Otros & Otros & Otros \\
\hline
\end{tabular}

Notas: (*) Con contrato fijo y remuneración con deposito fijo u otros medios; (*) Sin contrato fijo, con y sin remuneración por deposito u otra fuente de pago (con y sin remuneración de horas extras); (\#) Sujeto sin contrato fijo y con cierto porcentaje de inversión en el negocio; (\#\#) Sujeto con inversión y sin inversión acorde a las necesidades del negocio.

Tabla 2.

Distribución de la muestra según Institución Educativa Superior

\begin{tabular}{|c|c|c|c|}
\hline Tipo de Institución & $\begin{array}{l}\text { Cantidad de } \\
\text { instituciones } \\
\text { participantes }\end{array}$ & $\begin{array}{c}\text { Distritos de } \\
\text { procedencia de la } \\
\text { institución }\end{array}$ & $\begin{array}{l}\text { Cantidad de } \\
\text { submuestra }\end{array}$ \\
\hline $\begin{array}{l}\text { Centro de Educación Técnica } \\
\text { Productiva }\end{array}$ & 5 & $\begin{array}{l}\text { San Juan } \\
\text { Lurigancho } \\
\text { Puente Piedra } \\
\text { Comas } \\
\text { Los Olivos } \\
\text { Ventanilla }\end{array}$ & 75 \\
\hline Instituto Pedagógico Superior & 4 & $\begin{array}{l}\text { Puente Piedra } \\
\text { San Juan } \\
\text { Lurigancho } \\
\text { Los Olivos } \\
\text { Ventanilla } \\
\end{array}$ & 38 \\
\hline $\begin{array}{l}\text { Instituto de Formación } \\
\text { Técnica profesional }\end{array}$ & 5 & $\begin{array}{l}\text { Comas } \\
\text { San Juan } \\
\text { Lurigancho } \\
\text { San Martín de Porres } \\
\text { Los Olivos } \\
\text { Ventanilla }\end{array}$ & 62 \\
\hline
\end{tabular}




\begin{tabular}{lclcc}
\hline Universidad Privada & 5 & $\begin{array}{l}\text { Los Olivos } \\
\text { San Juan de } \\
\text { Lurigancho }\end{array}$ & 69 \\
\hline Universidad Pública & 1 & Ventanilla & 41 \\
\hline
\end{tabular}

Fuente: Base de datos de la investigación

Nota: Total de la muestra $=285$ estudiantes.

El grupo de trabajadores iniciados también fueron incluidos en el estudio, ya que muchos de ellos eran individuos provenientes de provincia, los cuales migraron a Lima con el fin de culminar sus estudios de nivel secundario o primario. En cuanto al estudio, a todos los participantes se les repartió la hoja de consentimiento informado para dar conformidad a la evaluación como parte del procedimiento de selección. Si el sujeto se negaba a complementar la ficha entonces se le excluyó del estudio.

\section{Instrumentos}

Escala de Comportamiento Proactivo en las Organizaciones - ECPO (Wegener \& Fabrigar, 2004 en Kamia \& Porto, 2009) es una escala con 29 ítems en su versión original de tipo politómica con opción de cinco respuestas para cada una, orientada a la evaluación de la planificación de las acciones, oportunidad de cambio y enfrentamiento. Esta escala se adaptó al idioma español desde el portugués adaptado por Kamia y Porto en su estudio. La validez de contenido permitió discernir que dos ítems impedían recoger la respuesta clara de los participantes, por tal razón se excluyeron por sugerencia en la votación de expertos (vot. $<45 \%$ ), todos los ítems restantes conservaron su carácter semántico (acuerdo de aceptación > $92 \%$ ). El análisis de fiabilidad arrojó 0.864 de índice Alfa de Cronbach $(n=99)$, después del plan de pilotaje.

Escala de resiliencia - ER (Wagnild \& Young, 1993), es una escala de tipo politómica que evalúa las dimensiones ecuanimidad, sentirse bien solo, confianza, perseverancia y satisfacción; la cual está compuesta por 25 ítems con siete opciones de respuesta. El criterio de jueces permitió utilizar el total de ítems (acuerdo de aceptación > $95 \%)$, y el plan piloto $(n=99)$, remitió datos confiables en cuanto a su contenido $(\alpha=, 82)$.

\section{Procedimiento}

En un principio, se realizó un sondeo inicial de los emprendedores durante seis meses, se generó un listado general de conformación de grupos y así calcular la cantidad del público objetivo con las características necesarias (tabla 1), y evaluar el costo que demandaría la aplicación de los instrumentos. Debido a esto se recurrió a elaborar un plan que incluyera a todo posible participante en actividades masificadoras, entonces, se decidió contactarlos por segunda vez. Se invitó a los participantes a una actividad de Capacitación gratuita durante tres meses; esta se brindó por medio virtual, y en otras oportunidades en locales céntricos a sus centros de trabajo y de manera discontinua. En dichas oportunidades de reunión se pasó un instrumento previo, llamado "Clasificador", con el fin de ayudar a los investigadores en la selección de los participantes sobre su formación profesional y otros aspectos necesarios para el estudio como: a) Sufrimiento de perdida parental en la niñez, b) Separación de padres o tutor de modo legal o por rapto, c) Penuria económica antes y durante la formación profesional, d) Grado de necesidad económica, d) Grado de necesidad parental, e) Apoyo de tutores, f) Necesidad económica para fortalecimiento como emprendedor, g) Narración de pérdidas de recursos y materiales de trabajo.

Luego de un tiempo, se analizaron los datos brindados por los futuros participantes y se recibieron 307 propuestas de las cuales se eliminaron las no pertinentes, por lo que quedaron 285 estudiantes como sujetos de inclusión en el estudio. Luego se les contactó previamente para la aplicación de los instrumentos, lo cual se realizó durante tres meses en una visita a sus propios establecimientos o centros laborales por cada distrito, como recompensa se le otorgó a cada uno 
un souvenir y $\mathrm{Cd}$ con materiales académicos digitales de la Capacitación gratuita otorgada inicialmente. Finalmente, se tabularon los datos en la base de datos SPSS 22 para su análisis.

\section{Resultados}

\section{Resiliencia y proactividad en estudiantes emprendedores según el apoyo familiar}

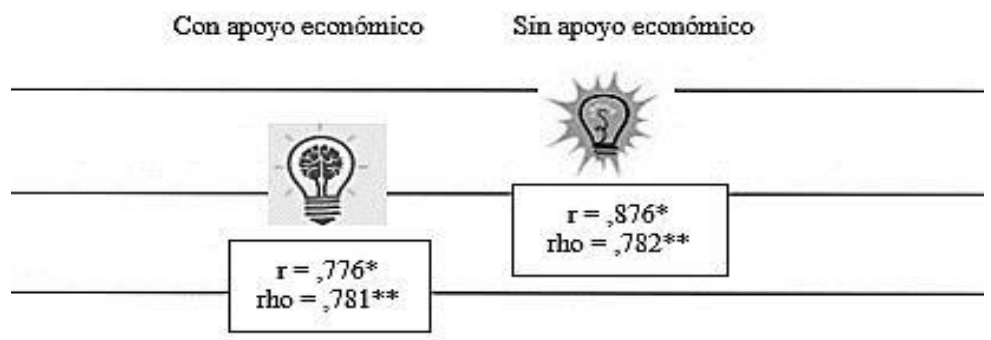

Figura 1. Relación entre resiliencia y proactividad de acuerdo con el apoyo familiar.

Notas: $* \mathrm{p}<.001 ; * * \mathrm{p}<.005 ; \mathrm{f}($ con apoyo económico $)=163 ; \mathrm{f}(\sin$ apoyo económico $)=122$.

\section{Resiliencia y proactividad por distritos de Lima.}

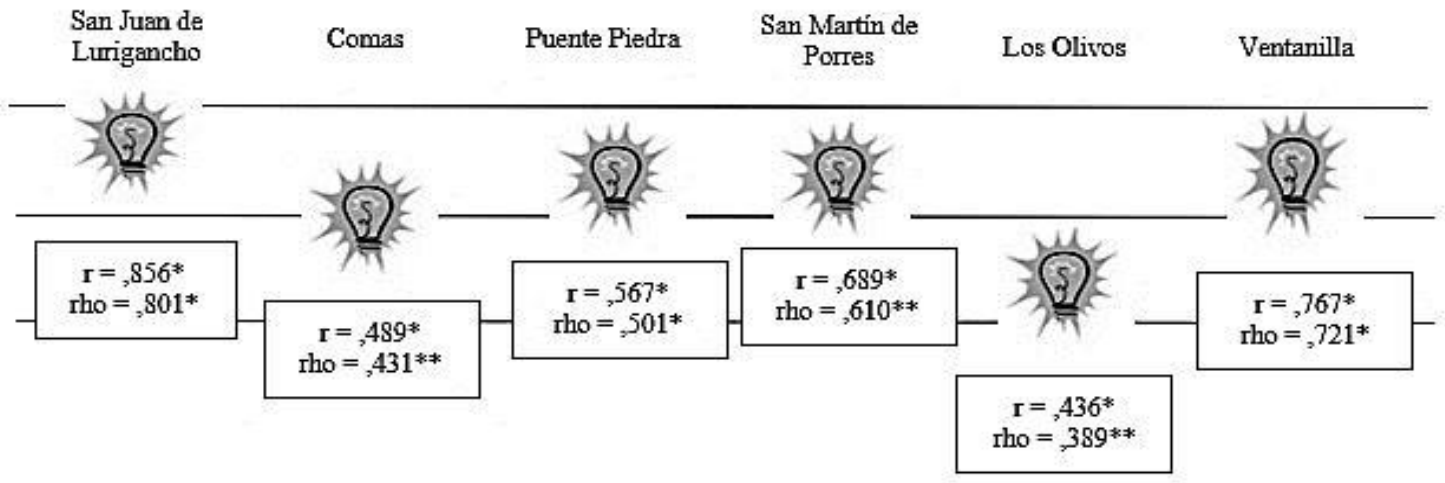

Figura 2. Relación entre resiliencia y proactividad en emprendedores de Lima.

Notas: $* \mathrm{p}<.001 ; * * \mathrm{p}<.005$. 


\section{Diferencias en proactividad y resiliencia por tipo de emprendedor.}

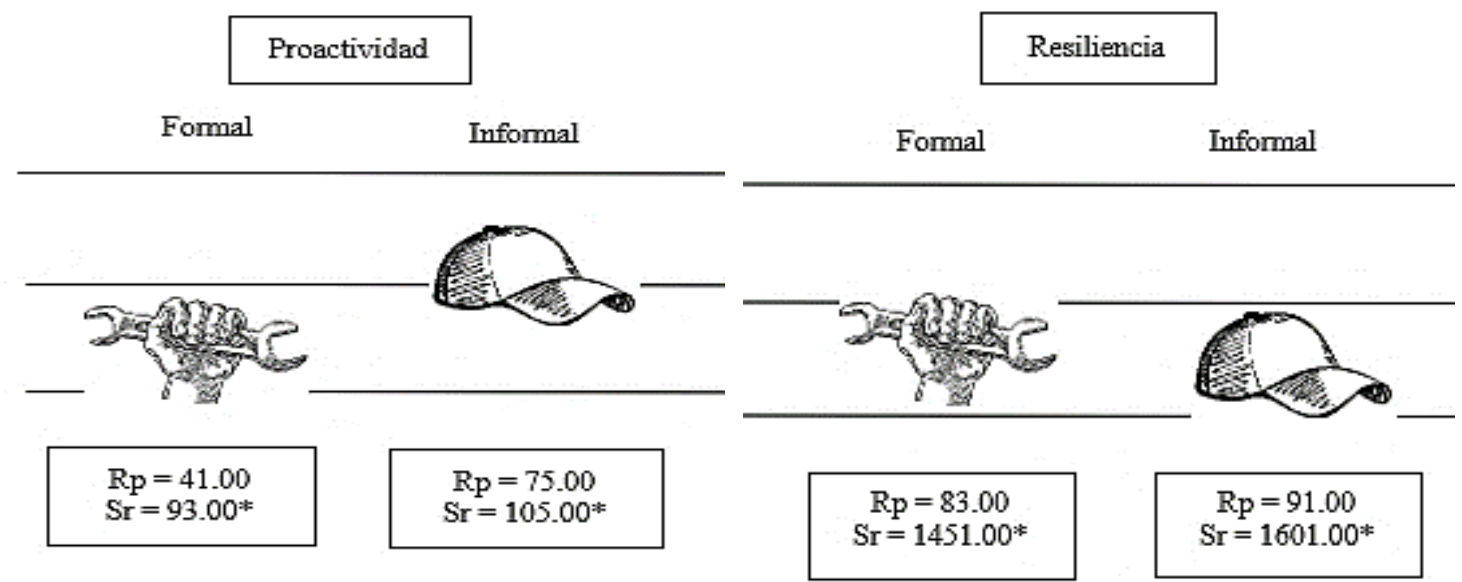

Figura 3. Diferencias de proactividad entre emprendedores formales e informales.

Notas: $* \mathrm{U}=201.000 ; \mathrm{p}<.001$.
Figura 4. Diferencias de resiliencia entre emprendedores formales e informales. Notas: * $\mathrm{U}=322.000 ; \mathrm{p}>.001$.

\section{Discusión}

\section{Estudiantes emprendedores con y sin apoyo familiar}

Los estudiantes emprendedores que no perciben apoyo económico familiar suelen desarrollar mayor resiliencia y proactividad en sus puestos laborales mientras prosiguen con sus estudios superiores, a diferencia de aquellos estudiantes que con apoyo económico de su familia o de algún tutor subsisten en la carrera profesional adoptada. Esto se comprobó en los hallazgos paramétricos y no paramétricos que respaldaron la correlación entre la resiliencia y proactividad del grupo de estudiantes que desarrollaron una inversión emprendedora durante el tiempo que también realizaron su formación profesional (figura 1), aunque se notó mayor correlación en los sujetos que no recibieron apoyo económico durante su profesionalización; es importante notar que es importante el apoyo o soporte que dicta la compañía en el trascurso de la formación profesional, y así lo ha sido para el $46 \%$ de sujetos que logró ser resiliente y proactivo bajo la compañía de sus familiares, y en el $54 \%$ de estudiantes en los que se presentó dicha relación pero sin estar acompañados de sus familiares, o en otras palabras, en aquellos que se forjan de forma independiente del seno familiar.

En este último grupo, los factores encontrados por Kamia y Porto (2009): búsqueda de oportunidades de cambio, planificación y ejecución de ideas y enfrentamiento a obstáculos (p. 363), han funcionado como factores más amplios que los de evaluación, imaginación y autoconciencia brindados inicialmente por Covey (1996) y los de Dehnad (2017), y en la actualidad algunas influencias de tipo económico, personal emocional, personal social determinan la fortaleza de los jóvenes emprendedores independientes como en otros estudios donde el estudiante debe auto dominarse (Bradshaw et al., 2018; Keskinkilic-Kara \& Zafer-Gunes, 2017); por esto, ante distintas dificultades y adaptaciones al cambio, este grupo de sujetos ha resultado más práctico para la superación y dificultad académica (Fernandez-Ronquillo et al., 2018; Lámbarry et al., 2016; Salessi \& Omar, 2017); ya que son resilientes como en muestras en que necesitan superar desde la aceptación de la verdad ante el obstáculo con el mismo pragmatismo que para ser proactivos (Didkowsky et al., 2013; Ungar \& Liebenberg, 2010; Ungar, 2013; 2014). 
El grupo de estudiantes resilientes y proactivos que suelen ser apoyados por sus padres también presentó alto nivel de correlación en el estudio, pero a diferencia de aquellos que no contemplan esta opción, mantienen el apoyo que fortalece la capacidad resiliente y proactiva ante el crecimiento profesional y que es brindado por sus padres u otros acompañantes que hacen el papel de tutor (Adjei et al., 2017; Ávalo et al., 2016; Brouskeli et al., 2018; Chen, 2016; Dutra-Thomé et al., 2016; Olea-Miranda et al., 2016; Peraza et al., 2016; Redchenko, 2016; Torrente et al., 2013), estas fortalezas aportan en su pensamiento visionario para ser emprendedores, aunque no está comprobado si superarían pérdidas materiales o humanas durante su crecimiento emprendedor podrían seguir con el crecimiento profesional en paralelo.

\section{Proactividad y resiliencia en los distritos de Lima}

Las correlaciones entre proactividad y resiliencia de los estudiantes emprendedores de los distritos de San Juan de Lurigancho y Ventanilla son más fuertes del distrito Limeño, es decir, son más proactivos y a la vez, suelen incrementar su nivel de resiliencia conforme se forman en las instituciones de educación superior. Las correlaciones de coeficiente más bajo fueron las de Comas y Los Olivos; por último, las correlaciones en los jóvenes de otros distritos de Lima fueron moderadamente aceptables (figura 2), se describió que la correlación entre la proactividad y resiliencia más intensa fue la perteneciente a los jóvenes del distrito de San Juan de Lurigancho, secundándole la del grupo de Ventanilla; y esto es una respuesta clara y evidente que esta relación sustenta la única implicancia del apoyo familiar como para entender la relación, sino que también se atribuye a otros factores como el contexto, ya que ambos distritos suelen presentar mayores riesgos para los jóvenes estudiantes en este tipo de contextos, ya que habría que acotar que los evaluados pertenecían a asociaciones poblacionales de poca económica y alto nivel de otros factores como el pandillaje, robos y delincuencia juvenil.

Ante estos últimos factores, es importante decir que se presentaron valores porcentuales mayores al $90 \%$ en ambos distritos de alto nivel de resiliencia y proactividad. Aunque, se analizaron por muestras separadas, los estudiantes de ambos distritos presentaron puntuaciones altas en la respuesta a ambos cuestionarios, y eso fue un elemento necesario para comprender la capacidad emprendedora de ambos grupos. Esto les ha brindado perdurabilidad en el campo de acción emprendedora, lo que es similar a otros estudios que implican factores como remuneración, toma de decisiones y sostenibilidad (Caraballo, 2015; Ortiz, 2016; Polanco et al., 2016). Si se analiza desde el lado del factor de resiliencia, los jóvenes mediante sus acciones de inversión persiguen su visión, y a largo plazo se generan un marco de desarrollo personal aunado a la profesionalización, por lo tanto, esto responde a lo planteado por la teoría de la resiliencia por factores de interrelación social (Cyrulnik, 2016; Ungar, 2008; 2012; 2013; 2014; West, 2005), a través de la cual se evidencia que estos jóvenes persisten en alcanzar un desarrollo económico al inicio pero se transforma a algo más humano conforme pasan los meses y años, y progresan; en esto tienen mucho que ver las fortalezas del humano resiliente como la confianza en si mismo, ecuanimidad, perseverancia y la satisfacción personal.

\section{Proactividad y resiliencia en emprendedores formales e informales}

Los resultados obtenidos en dos grupos: a) Los estudiantes proactivos presentan diferencias remarcadas en cuanto a sus características laborales y personales, por lo analizado, es mayor la proactividad en los jóvenes emprendedores con tendencia a informalización; y b) Los estudiantes emprendedores presentan similar nivel de resiliencia en su proceso de emprendorismo, sin embargo, dicha resiliencia es de alto nivel en ambos grupos. Dicha descripción provino de la determinación estadística de mayor diferencia del valor proactivo que el resiliente en estudiantes emprendedores informales (dif. $(\mathrm{Rp})=34.00$; dif. $(\mathrm{Sr})=12.00)$, lo cual ha sido significativo en su formato estadístico. Sin embargo, las características de la comparación en la proactividad demostraron que los estudiantes que emprenden en mayor proporción algún negocio durante el recorrido de su formación profesional son más proactivos que los que lo realizan con formalidad. 
La proactividad en el grupo de emprendedores informales ha sido mayor porque estiman una mayor producción y venta al público objetivo interesado en lo que ellos ofrecen, por esto recurren a tácticas de inversión privada informal (De Souza y Machado, 2013; Olea-Miranda et al., 2016; Pennaforte, 2016; Polanco et al., 2016; Redchenko, 2016; Zarate-Hoyos y Larios-Meoño, 2015), con el fin de obtener recursos económicos al menor tiempo posible y así mantener sus estudios superiores (Bradshaw et al., 2018; Galiana, 2015). En cambio, en el análisis de resiliencia se obtuvieron índices similares en cuanto al grupo de los emprendedores informales y formales, sin embargo, sus índices fueron de alto nivel en ambos grupos, cabe señalar que más del $75 \%$ de estudiantes de cada grupo presentó una media superior al del grupo general tomado como muestra, lo que quiere decir que, la resiliencia permite discernir el poder de superación de obstáculos del estudiante durante su carrera profesional y emprendimiento, pero no permite discernir sobre la capacidad que les permite elegir entre la formalidad e informalidad, sino más bien, la mera situación emprendedora les sirve como una capacidad para superar diversos límites para su desarrollo, por lo que todo es superación, así esta implique ir en contra de las normas sociales o simplemente, no obedecer a requisitos establecidos para la manutención de un negocio en particular. Para culminar con este análisis, las diferencias en proactividad entre sujetos formales e informales fueron aceptadas de acuerdo al planteamiento hipotético que se comprobó de modo estadístico; en cambio, la hipótesis de diferencias para la resiliencia no pudo ser comprobada entre informales y formales, ya que las evidencias no presentaron significación en su comparación, por lo tanto, fueron idénticas entre ambos tipos de emprendedor.

La principal contribución fue constatar que los jóvenes que emprenden una transformación activa de su profesión son capaces de superar las dificultades encontradas en su emprendimiento, por lo que se aduce que el emprendimiento es global, en otras palabras, el joven que se educa en el nivel superior de estudios y emprende a través de cierta inversión económica se genera una visión más poderosa y duradera como parte de su sociedad.

Se descubrió que el factor contexto genera influencia en el estudiante emprendedor. Dicho factor conjetura el fortalecimiento del emprendedor resiliente y estudiante proactivo como en todo proceso de culturización en el que se desarrollen competencias humanas. Estas competencias se auto refuerzan entre sí durante su formación en la práctica emprendedora.

Por último, se verificó que la proactividad resulta más interactiva en los emprendedores de centros de trabajo informal que en los de tipo formal. Esto es similar a otros resultados de estudios que remitieron que el pensamiento visionario permite alcanzar logros profesionales en emprendedores a corto tanto como emprendedores a largo plazo.

El acceso multi-teórico para la evaluación de las distintas subpoblaciones, no ha permitido medir a sus integrantes por los rasgos más específicos equiparables a las dimensiones de proactividad (Covey, 1996; Dehnad, 2017; Xifra, 2009) y resiliencia (Cyrulnik, 2016; Ungar, $2008 ; 2012$ ) ya que cada teoría desprende distintas vertientes y cuya adaptabilidad al contexto limeño es complejo. Esto no permitió un análisis de relación dimensional, y así conocer con mayor especificidad a las diferentes muestras, por lo que también se cree que, al contar con instrumentos con relativa cercanía, mantenían una orientación hacia la vida empresarial por el lado proactivo, y otro, hacia el comportamiento resiliente.

En cuanto a la evaluación comparativa final, es necesario acotar que en los estudiantes podrían haber influido otros factores que determinaron su respuestas en cuanto a resiliencia, entre los cuales, el factor estrés y necesidad implicaron que discriminaran erróneamente estos factores relacionándolos con el estrés académico que toda universidad exige (Cabrera, 2015; Chafloque, et al. 2018), y en este caso también fue así ya que la muestra se compuso por estudiantes con distintos niveles cognitivos y a su vez, en resiliencia.

Otra limitación fue el origen diferenciado a nivel socioeconómico, y esto debido a que los estudiantes pertenecieron a distintos distritos en los cuales vivían y en el mayor de los casos también desarrollaban su actividad económica, lo cual también es comprensible como un factor de acceso a 
poblaciones más particulares, así como ha aparecido en otros estudios distintivos por el factores social, económico y cultural (Adjei et al., 2017; Bradshaw et al., 2018; Larson et al., 2018; Pennaforte, 2016; Salessi \& Omar, 2017) y que en cierto modo, el tiempo para el estudio no permitió discernir con mayor exactitud.

\section{Referencias}

Adjei, A., Dontoh, S., \& Baafi-Frimpong, S. (2017). College Climate and Teacher-Trainee's Academic Work in Selected Colleges of Education in the Ashanti Region of Ghana. Journal of Education and Practice, 8(9), 141-160. Recuperado de http://www.iiste.org/Journals/index.php/JEP/article/view/36069

Atoche, M. (2017). Factores limitantes del empleo para los jóvenes egresados del Programa Nacional de Empleo Juvenil Jóvenes Productivos, de la Provincia de Arequipa, año 2015 (tesis de maestría). Pontificia Universidad Católica del Perú, Lima, Perú.

Ávalo, J.A., Yagüe, J.L., \& Cangahuala, G. (2016). El capital social y la planificación adaptativa en una comunidad industrial innovadora del Perú. Estudios Gerenciales, 32, 162-169, doi: http://dx.doi.org/10.1016/j.estger.2016.05.001

Bello, L. (2013). Jovens negros e ensino superior no Brasil: desvantagens no acesso e processo deresiliencia. Identidade! São Leopoldo, 18(2), 214-228. Recuperado de http://www.periodicos.est.edu.br/index.php/identidade/article/view/1014

Bradshaw, C. P., Pas, E.T., Bottiani, J.H., Reinke, W., \& Rosenberg, M.S. (2018). Promoting Cultural Responsivity and Student Engagement Through Double Check Coaching of Classroom Teachers: An Efficacy Study. School Psychology Review, 47(2), 118-134. doi: http://dx.doi.org/10.17105/SPR-2017-0119.V47-2

Brouskeli, V., Kaltsi, V., \& Loumakou, M. (2018). Resilience and occupational well-being of secondary education teachers in Greece. Issues in Educational Research, 28(1), 43-60. Recuperado de http://www.iier.org.au/iier28/brouskeli.pdf

Cabrera, L. (2015). Efectos del proceso de Bologna en la reducción del abandono de estudios universitarios: datos para la reflexión y propuestas de mejora. Revista Fuentes, 16, 39-62, doi: http://dx.doi.org/10.12795/revistafuentes.2015.i16.02

Chafloque, R., Vara-Horna, A., Lopez-Ordar, D., Santi-Huaranca, I., Díaz-Rosillo, A., \& Asencios-Gonzalez, Z. (2018). Ausentismo, presentismo y rendimiento académico en estudiantes de universidades peruanas. Propósitos y Representaciones, 6(1), 83-133. doi: http://dx.doi.org/10.20511/pyr2018.v6n1.177

Caraballo, M. (2015). Análisis de los factores que determinan la rentabilidad de las microempresas en el área sur de Puerto Rico para el 2012 (Tesis doctoral), Universidad de Turabo, Puerto Rico.

Chen, C. (2016). The Role of Resilience and Coping Styles in Subjective Well-Being Among Chinese University Students. The Asia-Pacific Education Researcher, 25(3), 377-387. doi: https://doi.org/10.1007/s40299-016-0274-5

Covey, S. (1996). Los 7 hábitos de la gente altamente efectiva. Barcelona: Paidós Ibérica.

Covey, S. (2007). Los 7 hábitos de los adolescentes altamente efectivos. México D.F.: Grijalbo.

De Souza, M.T., \& Machado, I.M. (2013). Empreendedorismo e resiliência: mapeamento das competências técnicas e comportamentais exigidas na atualidade. Revista de pensamento \& Realidade, 28(2), 59-76. Recuperado de https://revistas.pucsp.br/index.php/pensamentorealidade/article/view/16430

Dehnad, V. (2017). A Proactive Model to Control Reactive Behaviors. World Journal of Education, 7(4), 24-31. doi: https://doi.org/10.5430/wje.v7n4p24

Didkowsky N., Ungar M., \& Liebenberg L. (2010). Using Visual Methods to Capture Embedded Processes of Resilience for Youth across Cultures and Contexts, J Can Acad Child Adolesc Psychiatry, 19(1), 12-18, https://europepmc.org/articles/pmc2809441

Dutrá-Thomé, L., Siqueira, A., \& Koller, S. H. (2016). O Desafio de Conciliar Trabalho e Escola: Características Sociodemográficas de Jovens Trabalhadores e Não-trabalhadores. Psicologia: Teoria e Pesquisa, 32(1), 101-109. doi: http://dx.doi.org/10.1590/010237722016011944101109 
Faúndez, R., Labarca, J.P., Cornejo, M.F., Villarroel, M., \& Gil, F.J. (2017). Ranking 850, transición a la educación terciaria de estudiantes con desempeño educativo superior y puntaje PSU insuficiente. Pensamiento educativo. Revista de investigación Educacional Latinoamericana, 54(1), 1-11, doi: https://doi.org/10.7764/PEL.54.1.2017.2

Fernandez-Ronquillo, M.A., Llinas-Audet, X., \& Ferran-Sabate. (2018). Modelo de competencias para microempresarios como instrumento de desarrollo productivo. Revista Espacios, 39(7). Recuperado de http://hdl.handle.net/2117/116095

Fletcher, D., \& Sarkar, M. (2013). Psychological resilience: A review and critique of definitions, concepts, and theory. European Psychologist, 18, 12-23. https://doi.org/10.1027/10169040/a000124

Galiana, D.R. (2015). Análisis de la felicidad, resiliencia y optimismo como factores emocionales en la inserción laboral de los universitarios (tesis doctoral). Universidad Miguel Hernández De Elche, España.

Garrosa-Hernández, E., Carmona-Cobo, I., Ladstatter, F., Blanco, L.M., \& Cooper-Thomas, H.D. (2013). The relationships between family-work interaction, job-related exhaustion, detachment, and meaning in life: A day-level study of emotional well-being. Journal of Work and Organizational Psychology 29, 169-177. doi: http://dx.doi.org/10.5093/tr2013a23

Gaxiola, J.C., González, S., Domínguez, M., Gaxiola, E. (2013). Autorregulación, metas y rendimiento académico en bachilleres con disposiciones resilientes y no resilientes. Revista Interamericana de Psicología/Interamerican Journal of Psychology, 47(1), 7182. doi: https://doi.org/10.30849/rip/ijp.v47i1.203

Geertshuis, S., Jung, M., \& Cooper-Thomas, H. (2014). Preparing Students for Higher Education: The Role of Proactivity. International Journal of Teaching and Learning in Higher Education 26(2), 157-169. $\quad$ Recuperado de http://www.isetl.org/ijtlhe/abstract.cfm?mid=1689

Guevara, S. (2017). Resiliencia y Satisfacción con la Vida en estudiantes de la Universidad Nacional de Educación "Enrique Guzmán y Valle" (tesis de maestría), Universidad Nacional Enrique Guzmán y Valle, Perú.

Hernández, R., Fernández, C., \& Baptista, P. (2014). Metodología de la investigación (6º ed.). McGraw-Hill Education: México D.F.

Holguin, J.A. (2017). Efectos de conductas proactivas y prosociales en incidentes críticos de escolares limeños. Propósitos y representaciones, 5(2), 185244, doi: http://dx.doi.org/10.20511/pyr2017.v5n2.172

Instituto Nacional de Estadística e Informática - INEI (2017). Homicidios en el Perú. Contándolos uno a uno, 2011 - 2016, informe estadístico $N^{\circ}$ 5. Comité estadístico interinstitucional de la criminalidad - CEIC: Lima.

Kamia, M., \& Porto, J.B. (2009). Desenvolvimento e validação da escala de comportamento proativo nas organizações - ECPO. Avaliação Psicológica, 8(3), 359-367. Recuperado de http://pepsic.bvsalud.org/pdf/avp/v8n3/v8n3a08.pdf

Keskinkilic-Kara, S.B., \& Zafer-Gunes, D. (2017). Relationship Between Implicit Leadership and Proactive Behaviors of School Principals. Journal of Education and Practice, 8(3), 78-88. Recuperado de: https://www.iiste.org/Journals/index.php/JEP/article/view/35100

Lámbarry, F., Trujillo, M.M., \& Cumbres, C.G. (2016). Stress from an administrative perspective in public transport drivers in Mexico City: Minibus and metrobus. Estudios gerenciales, 32, 112-119. Doi: http://dx.doi.org/10.1016/j.estger.2016.02.003

Larson, K.E., Pas, E.T., Bradshaw, C.P., Rosenberg, M.S., \& Day-Vines, N.L. (2018). Examining How Proactive Management and Culturally Responsive Teaching Relate to Student Behavior: Implications for Measurement and Practice. School Psychology Review, 47(2), doi:http://dx.doi.org/10.17105/SPR-20170070.V47-2

Moreno-Jiménez, B., \& Gálvez, M. (2013). El efecto del distanciamiento psicológico del trabajo en el bienestar y la satisfacción con la vida: un estudio longitudinal. Journal of Work and Organizational Psychology, 29, 145-151. doi: http://dx.doi.org/10.5093/tr2013a20 
Olea-Miranda, J., Contreras, O.F., \& Barco-Valenzuela, M. (2016). Las capacidades de absorción del conocimiento como ventajas competitivas para la inserción de pymes en cadenas globales de valor. Estudios Gerenciales, 32, 127-136, doi: http://dx.doi.org/10.1016/j.estger.2016.04.002

Ortiz, M.A. (2016). Factores explicativos del fracaso y del éxito en las microempresas de la República Dominicana: un estudio empírico (tesis doctoral). Universidad Politécnica de Cartagena, Cartagena, España.

Ozmusul, M. (2017). We Need Resilient School Leaders in the face of Chaos and Complexity. Education Reform Journal 2(2), 17-25. doi: https://doi.org/10.22596/erj2017.0201.17.25

Peraza, E.H., Gómez, J.M., \& Aleixandre, G. (2016). Los factores determinantes del comportamiento innovador de las cooperativas: un análisis para el caso de Castilla y León. Revesco, 122, 252-284, doi: http://dx.doi.org/10.5209/rev_REVE.2016.v122.52025

Pereira, E.M.F. (2016). Vinculação, autocompaixão e Resiliência em jovens adultos: Relações com as competências sociais (Tesis de maestría). Universidade Lusófona de Humanidades e Tecnologias, Lisboa, Portugal.

Polanco, J., Ramírez, F., \& Orozco, M. (2016). Incidencia de estándares internacionales en la sostenibilidad corporativa: una perspectiva de la alta dirección. Estudios Gerenciales, 32, 181-192, doi: http://dx.doi.org/10.1016/j.estger.2016.05.002

Pennaforte, A. (2017). The influence of proactive socialization behaviors and team socialization on individual performance in the team. Asia-Pacific Journal of Cooperative Education, 17(4), 413-421. Recuperado de https://files.eric.ed.gov/fulltext/EJ1131581.pdf

Price, D.V., \& Sedlak, W. (2018). Creating opportunity for all. Building pathways from continuing Education to credit programs. Equal Mesure january 2018, Achieving the Dream, http://www.achievingthedream.org/

Quijada, K.Y. (2018). Rasgos de la excelencia en la investigación: proactividad, pasión por el conocimiento y resiliencia. Sinéctica, 51(2), 1-19, Recuperado de https://sinectica.iteso.mx/index.php/SINECTICA/article/view/877

Redchenko, N.N. (2016). Interdisciplinary Learning as a Basis for Formation of Intercultural Communicative Competence. International Journal of Environmental \& Science Education, 11(13), 6195-6202. Recuperado de http://www.ijese.net/makale/787

Sabino, C. (2014). El proceso de investigación. Episteme: Guatemala.

Salessi S., \& Omar, A. (2017). Comportamientos proactivos en el trabajo: una puesta al día. Revista Argentina de Ciencias del Comportamiento, 9(3), 82-103. doi: http://dx.doi.org/10.32348/1852.4206.v9.n3.17800

Silva, Y.M., \& Jiménez, A. (2015). Estudiantes de contextos vulnerables en una universidad de élite. Revista de Educación Superior, 3(175), 95-119. Recuperado de http://publicaciones.anuies.mx/revista/175/3/4/es/estudiantes-de-contextos-vulnerablesen-una-universidad-de-elite

Simón J. (2017). Egresados de nuevas carreras universitarias: competir desigualmente en el mercado de trabajo. Propósitos y Representaciones, 5(1), 129203 doi: http://dx.doi.org/10.20511/pyr2017.v5n1.146

Stanley, N., Nguyen, K., Wilson, H., Stanley, L., Rank, A., \& Wang, Y. (2015). Storytelling, Values and Perceived Resilience among Chinese, Vietnamese, American and German Prospective Teachers. Universal Journalof Educational Research, 3(8), 520-529. doi: http://dx.doi.org/10.13189/ujer.2015.030807

Superintendencia Nacional de Aduanas y de Administración Tributaria-SUNAT (2018). Buscador Mypes (Visualizado: 27/08/2018). Recuperado de: http://www.sunat.gob.pe/orientacion/mypes/caracteristicas-microPequenaEmpresa.html

Torrente, P., Salanova, M., \& Llorens, S. (2013). Spreading engagement: On the role of similarity in the positive contagion of team work engagement. Journal of Work and Organizational Psychology, 29(3), 153-159. doi: http://dx.doi.org/10.5093/tr2013a21

Ungar, M. (2008). Resilience across Cultures. British Journal of Social Work, 38(2), 218-235. doi: https://doi.org/10.1093/bjsw/bcl343 
Ungar, M. (2012). Social Ecologies and Their Contribution to Resilience. In: Michael Ungar (editor). The Social Ecology of Resilience. A Handbook of Theory and Practice. Springer: New York, 13-31, http://psycnet.apa.org/record/2011-30122-002

Ungar, M. (2014). Practitioner Review: Diagnosing childhood resilience - a systemic approach to the diagnosis of adaptation in adverse social and physical ecologies. The Journal of child Psychology and Psychiatry, 56(1), 4-17. doi: https://doi.org/10.1111/jcpp.12306

Ungar, M., Liebenberg, L., Dudding, P., Armstrong, M., \& van de Vijver, F.J.R. (2013). Patterns of service use, individual and contextual risk factors, and resilience among adolescents using multiple psychosocial services. Child Abuse \& Neglect, 32 (23), 150-159. doi: https://doi.org/10.1016/j.chiabu.2012.05.007

Velásquez-Acosta, P. (2017). Medida de la Resiliencia en estudiantes universitarios de primer ciclo de la Escuela Profesional de Administración y Negocios Internacionales de la Facultad de Ciencias Empresariales de la Universidad Alas Peruanas (Tesis de Maestría). Universidad de Piura. Piura, Perú.

Wagnild, G., \& Young, H. (1993). Development and Psychometric Evaluation of the Resilience Scale. Journal of Nursing Measurement, 1(2), 165-177. Recuperado de https://www.ncbi.nlm.nih.gov/pubmed/7850498

West, J. (2005). Lessons learned from poor African American youth. Resilient strenghts in coping with adverse environments. In: Michael Ungar (editor). Handbook for working with children and youth. Pathways to resilience across cultures and contexts. SAGE: California, London, New Dehli, 45-56. Xifra, J. (2009). Comunicación proactiva. La gestión de conflictos potenciales en las organizaciones. Barcelona: Gedisa.

Zarate-Hoyos, G.A., \& Larios-Meoño, F. (2015). El rol de las universidades y otras instituciones en el emprendimiento exi-toso: visiones de una revisión de la literatura. Propósitos y Representaciones, 3(2), 261-317. doi: http://dx.doi.org/10.20511/pyr2015.v3n2.82 